\title{
ANALYSIS OF REDUCTION IN COMPLEXITY OF MULTIPLE INPUT- MULTIPLE OUTPUT-ORTHOGONAL FREQUENCY DIVISION MULTIPLEXING SYSTEMS WITH CARRIER FREQUENCY OFFSET ESTIMATION AND CORRECTION
}

\author{
Sabitha Gauni and Kumar Ramamoorthy \\ Department of Electronics and Communication Engineering, \\ Faculty of Engineering and Technology, SRM University, Kattankulathur, India
}

Received 2013-09-10; Revised 2013-09-19; Accepted 2013-11-13

\begin{abstract}
Orthogonal Frequency Division Multiplexing (OFDM) is a promising research area in Wireless Communication for high data rates. The Multiple Input-Multiple Output (MIMO) technology when incorporated with the OFDM system promise a significant boost in the performance. But, the MIMOOFDM systems are very sensitive to Carrier Frequency Offset (CFO) as it deteriorates the system performance with the rise of Inter-Carrier-Interference (ICI). A theoretical analysis to evaluate the performance of Orthogonal Frequency Division Multiplexing (OFDM) systems is done here, under the combined influence of phase offset and frequency offset over rayleigh, Weibull and Nakagami fading channels using Binary Phase Shift Keying (BPSK) Modulation. The analysis of increase in Bit Error Rate (BER) caused by the presence of phase offset and frequency offset is evaluated assuming Gaussian probability density function. Hence the estimation and correction of CFO plays a vital role in MIMOOFDM systems. A method for CFO estimation and correction is analyzed in the MIMO-OFDM system with 16-QAM modulation. In non-pilot-aided systems the CFO acquisition is done using Maximum Likelihood Estimation (MLE) algorithm. The proposed scheme uses the same block for CFO correction of MIMOOFDM symbols. Since the same phase calculation block is used for the ML estimation along with the acquisition, the computational cost and the complexity of implementation is reduced.
\end{abstract}

Keywords: Carrier Frequency Offset (CFO), Orthogonal Frequency Division Multiplexing (OFDM), Maximum Likelihood Estimation (MLE), Multiple Input Multiple Output (MIMO), Additive White Gaussian Noise (AWGN)

\section{INTRODUCTION}

Orthogonal Frequency Division Multiplexing (OFDM) is used in order to achieve high data rate in Wireless transmission. The use of multiple antennas at both the transmitter and receiver offers higher data rates compared to single antenna systems. The system capacity can be enhanced by the use of antenna arrays at the transmitter and receiver in rich scattering environments and at sufficiently high Signal to Noise Ratios (SNR). This results in Multiple-Input MultipleOutput (MIMO) configuration, which results in increasing the diversity gain on time-variant and frequency-selective channels. The features like robustness against multi-path fading, high spectral efficiency, has made OFDM more popular than the conventional single Technology, SRM University, Kattankulathur, India 
carrier systems. The MIMO techniques aims to improve the power efficiency by maximizing spatial diversity using Space-time block codes.

MIMO OFDM systems are also sensitive to Carrier Frequency Offset (CFO), due to the Doppler shifts or unmatched oscillators and Inter-Carrier-Interference (ICI).

The study deals with a new frequency offset correction scheme for MIMO-OFDM systems. The frequency acquisition is performed by first estimating the frequency offset using some simple comparisons and then the phase compensation is done in the frequency domain. After simple comparisons in the frequency domain, the proposed scheme makes correction to OFDM symbols and compensates using MLE algorithm. MLE algorithm is considered for the frequency correction scheme in the study as it is a highly efficient non-data-aided synchronization method. It shares a common block with the frequency tracking algorithm. The computational cost and implementation complexity is reduced to a great extent, by sharing the same phase calculation block with the acquisition step. This scheme does not require any pilot symbol as it uses the information brought by OFDM symbols; hence high bandwidth efficiency can be achieved.

Gauni et al. (2012) analyzed the OFDM system with Phase and frequency offsets.

Qatawneh (2013) compared the different modulation types based MIMO systems.

Ullah et al. (2012) proposed the design of transmitter using LS code of length 8190 bits which can measure multipath delay of minimum $0.13 \quad \hat{\mathrm{I}} 1 / 4 \mathrm{~s}$ and maximum $520 \hat{\mathrm{I}}^{1} / 4 \mathrm{~s}$.

Ahmed et al. (2010) presented the maximum likelihood estimation, Bayesian using Jeffrey prior and the extension of Jeffrey prior information for estimating the parameters of Weibull distribution of life time.

Kumaran et al. (2013) analyzed the performance of OFDM based bidirectional relay network which employs Physical Layer Network Coding (PLNC) in the presence of phase noise.

Suresh et al. (2012) proposed a novel method to estimate fine symbol timing error for MB-OFDM based UWB system which will be suitable for MB-OFDM receivers for UWB positioning and UWB communication.

\section{OFDM SYSTEM MODEL}

Consider an OFDM system with $\mathrm{N}$ sub-carriers. Let $\mathrm{X}(\mathrm{k})$ be the $\mathrm{k}^{\text {th }}$ OFDM data block to be transmitted with $\mathrm{N}$ subcarriers. These data blocks are used to modulate $\mathrm{N}$ orthogonal subcarriers. Then, using IDFT input signal is modulated. The modulated signal is represented as given in Equation (1):

$$
\mathrm{X}(\mathrm{n})=\frac{1}{\mathrm{~N}} \sum_{\mathrm{k}=0}^{\mathrm{N}-1} \mathrm{X}(\mathrm{k}) \exp (\mathrm{i} 2 \pi \mathrm{kn} / \mathrm{N}) \forall \mathrm{n}=0,1, \ldots . \mathrm{N}-1
$$

Due to the multipath propagation, the signal can be reflected, diffracted or diffused depending on the nature of the wave and the obstacle. The channel impulse response of a multipath fading channel is modeled as. $|h(n)| e^{-j \theta(n)}$ Assuming that frequency synchronization is achieved at the receiver side, the received signal can be represented in the frequency domainis given by Equation (2):

$$
\mathrm{R}(\mathrm{k})=\mathrm{X}(\mathrm{k}) \mathrm{H}(\mathrm{k})+\mathrm{N}
$$

where, $\mathrm{N}$ is an independent identically distributed (i.i.d) complex Gaussian noise component with zero mean and unit variance. The amplitude of $\mathrm{H}(\mathrm{k})$ is modeled as a rayleigh, Weibull and Nakagami fading distribution with the corresponding PDFs.

\section{BER ANALYSIS IN THE PRESENCE OF OFFSETS}

Here BER analysis is discussed in the presence of phase and frequency offsets in detail.

\subsection{Carrier Frequency Offset}

The absolute value of the actual $\mathrm{CFO}, \mathrm{f}_{\varepsilon}$, is either fraction or an integer multiple of subcarrier spacing, $\Delta \mathrm{f}$, or the sum of them. If $f_{\varepsilon}$ is normalized to $\Delta f$, then the resulting normalized $\mathrm{CFO}$ of the channel can be generally expressed as in Equation (3):

$$
\varepsilon=\frac{\mathrm{f}_{\varepsilon}}{\Delta \mathrm{f}}=\delta+\epsilon
$$

where, $\delta$ is an integer and $|\in| \leq 0.5$, where:

$$
\varepsilon=\frac{\text { frequency offset }}{\text { subcarrier spacing }}
$$

The effect of an integer CFO on OFDM system is different from the influence of a fractional CFO. In the event that $\delta \neq 0$ and $\in=0$, symbols transmitted on a certain subcarrier, e.g., subcarrier k, will shift to another subcarrier $\mathrm{K}_{\delta}$, given by Equation (4):

$$
\mathrm{k} \delta=\mathrm{k}+\delta \bmod \mathrm{N}-1
$$


Since the focus is on the ICI effect, we consider the normalized CFO, $\varepsilon$ assuming that no ICI is caused by an integer CFO. The nature of $\varepsilon$ is considered to be a Gaussian process, statistically independent of the input signal with zero mean and variance $\sigma_{3}^{2}$. To obtain the probability of error, we consider the PDF of $\varepsilon$ as Equation (5):

$$
\mathrm{f}(\varepsilon)=\frac{1}{\sigma_{\varepsilon} \sqrt{2 \pi}} \exp \left(-\frac{\varepsilon^{2}}{2 \sigma_{\varepsilon}^{2}}\right) \forall \varepsilon>0 \text { and } \mathrm{f}(\varepsilon)=0 \text { for } \varepsilon<0
$$

\subsection{Fading Channels}

The effect of the wireless channel on the transmitted signals is multiplicative, where the multiplicative term is assumed to be a complex Gaussian random variable.

\subsection{Rayleigh Fading Channel}

If the channel coefficient has zero mean, then such a channel is considered as rayleigh fading since the absolute value of the received amplitude is a rayleigh random variable.

The PDF of the received signal amplitude in a rayleigh fading channel is given by Equation (6):

$$
\mathrm{f}(\mathrm{x})=\frac{\mathrm{x}}{\sigma^{2} \alpha} \exp \left(-\frac{\mathrm{x}^{2}}{2 \sigma^{2} \alpha^{2}}\right) \quad \forall \mathrm{x}>0
$$

where, $x$ has a rayleigh distribution, $\sigma^{2}$ is the variance of received signal amplitude and $\alpha$ is the fading parameter.

\subsection{Weibull Fading Channel}

The Weibull distribution is related to a number of other probability distributions; in particular, it interpolates between the exponential distribution $(\mathrm{k}=1)$ and the rayleigh distribution $(\mathrm{k}=2)$.

The PDF of the recovered signal in Weibull fading channel is given by Equation (7):

$$
f(x)=\frac{k}{\alpha \lambda}\left(\frac{x}{\alpha \lambda}\right)^{k-1} \exp \left(-\frac{x}{\alpha \lambda}\right)^{k} \forall x>0
$$

where, $\mathrm{k}>0$ is the shape parameter and $\lambda>0$ is the scale parameter of the distribution.

\subsection{Nakagami Fading Channel}

The Nakagami-m or Nakagami distribution is a probability distribution related to the Gamma distribution.
It has two parameters: A shape parameter, $\mu$ and a second parameter controlling spread, $\Omega$. The PDF of the received signal amplitude in a Nakagami fading channel is given as Equation (8):

$$
\mathrm{f}(\mathrm{x})=\frac{2 \mathrm{~m}^{\mathrm{m}}\left(\frac{\mathrm{x}}{\alpha}\right)^{2 \mathrm{~m}-1}}{\Gamma(\mathrm{m}) \Omega^{\mathrm{m}}} \exp \left(-\frac{\mathrm{m}\left(\frac{\mathrm{x}}{\alpha}\right)^{2}}{\Omega}\right) \forall \mathrm{x}>0
$$

In the Nakagami fading channel, when the fading parameter, $m=1$ this channel becomes a rayleigh fading channel. The analysis of the system performance with Nakagami fading model is usually more tractable than Rician fading.

\section{PERFORMANCE ANALYSIS OF OFDM SYSTEMS WITH FREQUENCY OFFSET}

\subsection{BER Expressions for Frequency Offset}

Due to spectral overlapping, OFDM systems are sensitive to frequency offset. The orthogonality among the subcarriers is destroyed due to frequency offset, resulting in ICI, this causes degradation in the system performance. Hence estimation of the frequency offset is necessary to improve the system performance.

The recovered signal, $\mathrm{d}_{\mathrm{k}}$ on subcarrier $\mathrm{k}$ is given by Equation (9):

$\mathrm{d}_{\mathrm{k}}=\mathrm{I}_{0} \mathrm{~S}_{\mathrm{k}}$

where, $I_{0}$ is the attenuation and phase rotation of the desiredsignal, $S_{k}$ is the $\mathrm{k}^{\text {th }}$ transmitted signal.

From (1), it is clear that the dominant signal on the $\mathrm{k}^{\text {th }}$ subcarrier is $I_{0} S_{k}$. Hence, the magnitude mean of the dominant signal on the $\mathrm{k}^{\text {th }}$ subcarrier is given by Equation (10):

$$
\mathrm{m}=\left|\mathrm{I}_{0} \mathrm{~S}_{\mathrm{k}}\right|=\left|\frac{\sin \pi \varepsilon}{\pi \varepsilon} \mathrm{e}^{\mathrm{j} \pi \varepsilon} \mathrm{S}_{\mathrm{k}}\right|=\sin \mathrm{c}(\varepsilon)
$$

where, $\varepsilon=\mathrm{T}_{\mathrm{s}} \Delta \mathrm{f} \in(-0.5,0.5)$ is the frequency offset normalized to subcarrier spacing. The expectation $\left|\mathrm{d}_{\mathrm{k}}\right|$ of is given as:

$$
\begin{aligned}
& E\left(\left|d_{k}\right|\right)=\int_{-\infty}^{\infty} x p_{\left|d_{k}\right|}(x) d x \\
& E\left(\left|d_{k}\right|\right)=\sqrt{\frac{2\left(1-\sin c^{2}(\varepsilon)\right)}{\pi}} \exp \left\{-\frac{\sin c^{2}(\varepsilon)}{2\left(1-\sin c^{2}(\varepsilon)\right)}\right\}-\operatorname{sinc}(\varepsilon)
\end{aligned}
$$


The Second moment $\left|d_{k}\right|$ of is given as:

$$
E\left(\left|d_{k}\right|^{2}\right)=\int_{-\infty}^{\infty} x^{2} p_{\left|d_{k}\right|}(x) d x=1
$$

The Variance $\left|\mathrm{d}_{\mathrm{k}}\right|$ of can be expressed as:

$$
\sigma_{w}^{2}=E\left(\left|d_{k}\right|^{2}\right)-E^{2}\left(\left|d_{k}\right|\right)
$$

i.e.:

$$
\sigma_{\mathrm{w}}^{2}=1-\left[\begin{array}{l}
\sqrt{\frac{2\left(1-\sin \mathrm{c}^{2}(\varepsilon)\right)}{\pi}} \exp \\
\left\{-\frac{\sin \mathrm{c}^{2}(\varepsilon)}{2\left(1-\sin \mathrm{c}^{2}(\varepsilon)\right)}\right\}-\sin \mathrm{c}(\varepsilon)
\end{array}\right]^{2}
$$

Based on Equation (11), the PDF of the output, can be approximated as a Gaussian mixture as:

$$
\mathrm{p}_{\left|\mathrm{d}_{\mathrm{k}}\right|}(\mathrm{x})=\left[\frac{1}{\sigma_{\mathrm{w}} \sqrt{2 \pi}} \mathrm{e}^{-(\mathrm{x}-\mathrm{m})^{2} / 2 \sigma_{\mathrm{w}}{ }^{2}}+\frac{1}{\sigma_{\mathrm{w}} \sqrt{2 \pi}} \mathrm{e}^{-(\mathrm{x}+\mathrm{m})^{2} / 2 \sigma_{\mathrm{w}}{ }^{2}}\right] \mathrm{u}(\mathrm{x})
$$

where, $\mathrm{u}(\mathrm{x})$ is the unit step function:

$P_{b}(\varepsilon)=\int_{0}^{\infty}\left[\begin{array}{l}\frac{1}{\sigma_{w} \sqrt{2 \pi}} e^{-(x-m)^{2} / 2 \sigma_{w}^{2}} \\ +\frac{1}{\sigma_{w} \sqrt{2 \pi}} e^{-(x+m)^{2} / 2 \sigma_{w}^{2}}\end{array}\right] f(x) d x$

where, $f(x)$ represents the PDF of various fading distributions and Equation (12) gives the BER conditioned on the normalized frequency offset, $\in$. The unconditional BER is then given as Equation (13):

$\mathrm{P}_{\mathrm{e}}=\int_{0 \in=-0.5}^{\infty} \int_{\left|\mathrm{d}_{\mathrm{k}}\right|}^{0.5}(\mathrm{x}) \mathrm{f}(\mathrm{x}) \mathrm{f}(\varepsilon) \mathrm{d} \varepsilon \mathrm{dx}$

\section{MIMO-OFDM SYSTEM MODEL}

A typical MIMO OFDM implementation with Q transmit antennas and $\mathrm{L}$ receive antennas, denoted as a Q*L system is shown in Fig. 1.

For single antenna system, i.e., SISO, the transmitted signal is represented by $\mathrm{s}(\mathrm{k})$ and $\mathrm{N}$-point
IFFT is used in the transmitter section. The data is converted to parallel streams from serial form after encoding and interleaving in order to reduce random and burst noise on the data signals. Then N-point IFFT is done on the data samples after some digital modulation schemes like Binary Phase Shift Keying (BPSK), Quadrature Amplitude Modulation (QAM). After IFFT, the last L data samples are added to the $\mathrm{N}$ data samples as Cyclic Prefix (CP) to generate one OFDM symbol $s_{n}$ of length $N+L$. Frames are built in the next step and sent through the channel. Then the received signal Equation (14), along with its frequency offset is expressed in the presence of Additive White Gaussian Noise (AWGN) as:

$\mathrm{r}(\mathrm{k})=\mathrm{s}(\mathrm{k}) \exp \left(\frac{\mathrm{j} 2 \pi \mathrm{k} \varepsilon}{\mathrm{N}}\right)+\mathrm{w}(\mathrm{k})$

where, $\mathrm{w}_{\mathrm{l}}(\mathrm{k})$ is the Additive White Gaussian Noise $(\mathrm{AWGN})$ on the $1^{\text {th }}$ receive antenna with zero mean and variance $\sigma_{1}^{2}, h_{i, l}$ is the channel gain between the $i^{\text {th }}$ transmit antenna and the $1^{\text {th }}$ receive antenna and $\varepsilon$ denotes the $\mathrm{CFO}$ normalized to the inter-carrier spacing.

The CFO estimation includes two steps: Acquisition and tracking. A range of CFO estimation can be increased in acquisition, whereas the estimation range is much smaller in tracking. With respect to the estimation accuracy, tracking is better than that of acquisition. After acquisition, the remaining $\mathrm{CFO}$ must be within the tracking range for the tracking algorithm to be precise.

\section{ML ESTIMATOR WITH PROPOSED BLOCK}

The MLE algorithm block as shown in Fig. 2 is used for frequency estimation and correction, due to its high efficiency in non-data aided synchronization. The left half of the figure stands for the process of MLE algorithm and the remaining part takes care of the frequency tracking.

The ML estimation for $\theta$ and $\varepsilon$ is done by maximizing the argument $\Lambda(\theta, \varepsilon)$ Equation (15) and under the assumption that $r$ is a jointly Gaussian vector and the loglikelihood function can be written as Equation (16-18):

$$
\begin{aligned}
& \Lambda(\theta, \varepsilon)=|\gamma(\theta)| \cos (2 \pi \varepsilon+\angle \gamma(\theta))-\rho \Phi(\theta) \\
& \gamma(\mathrm{m}) \underline{\Delta} \sum_{\mathrm{k}=\mathrm{m}}^{\mathrm{m}+\mathrm{L}-1} \mathrm{r}(\mathrm{k}) \mathrm{r}^{*}(\mathrm{k}+\mathrm{N})
\end{aligned}
$$



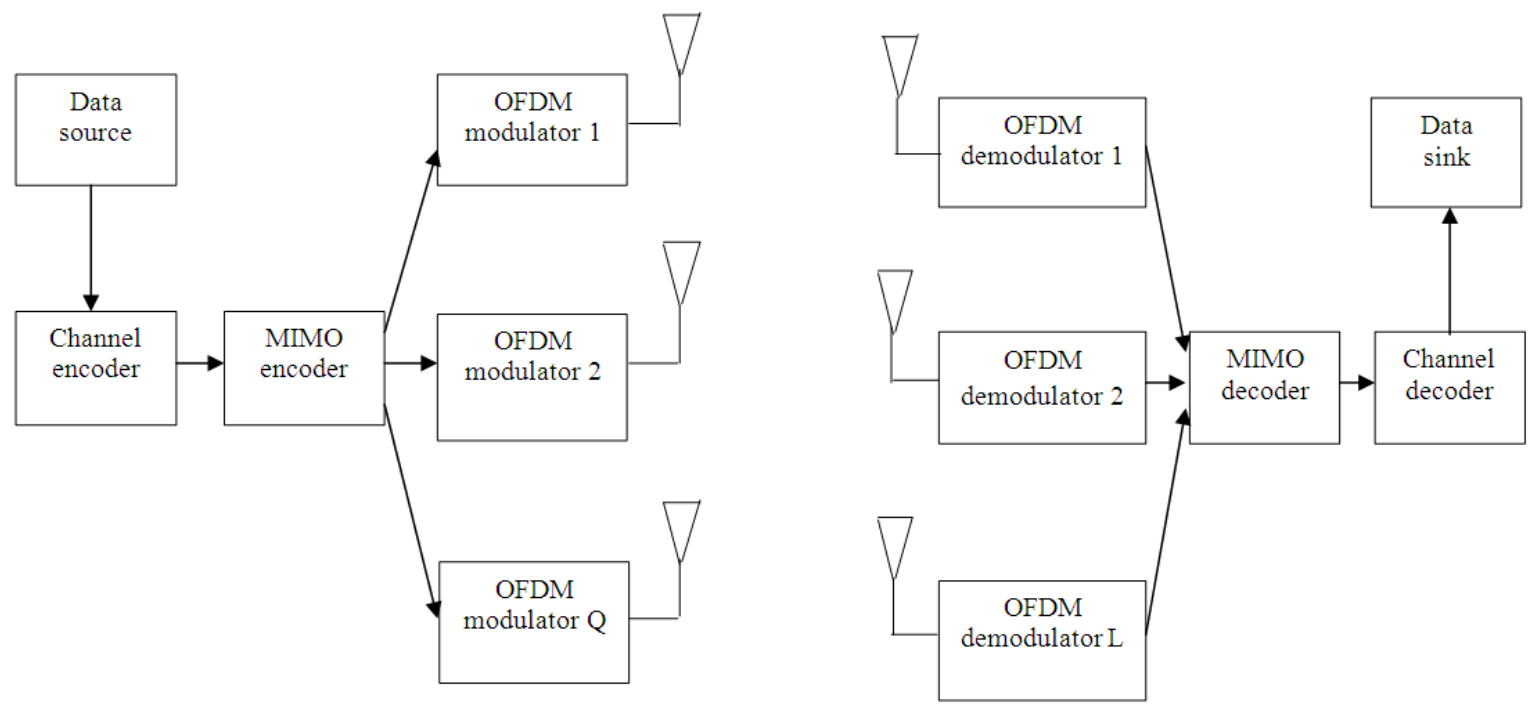

Fig. 1. MIMO-OFDM system with $\mathrm{Q}$ transmit antennas and $\mathrm{L}$ receive antennas

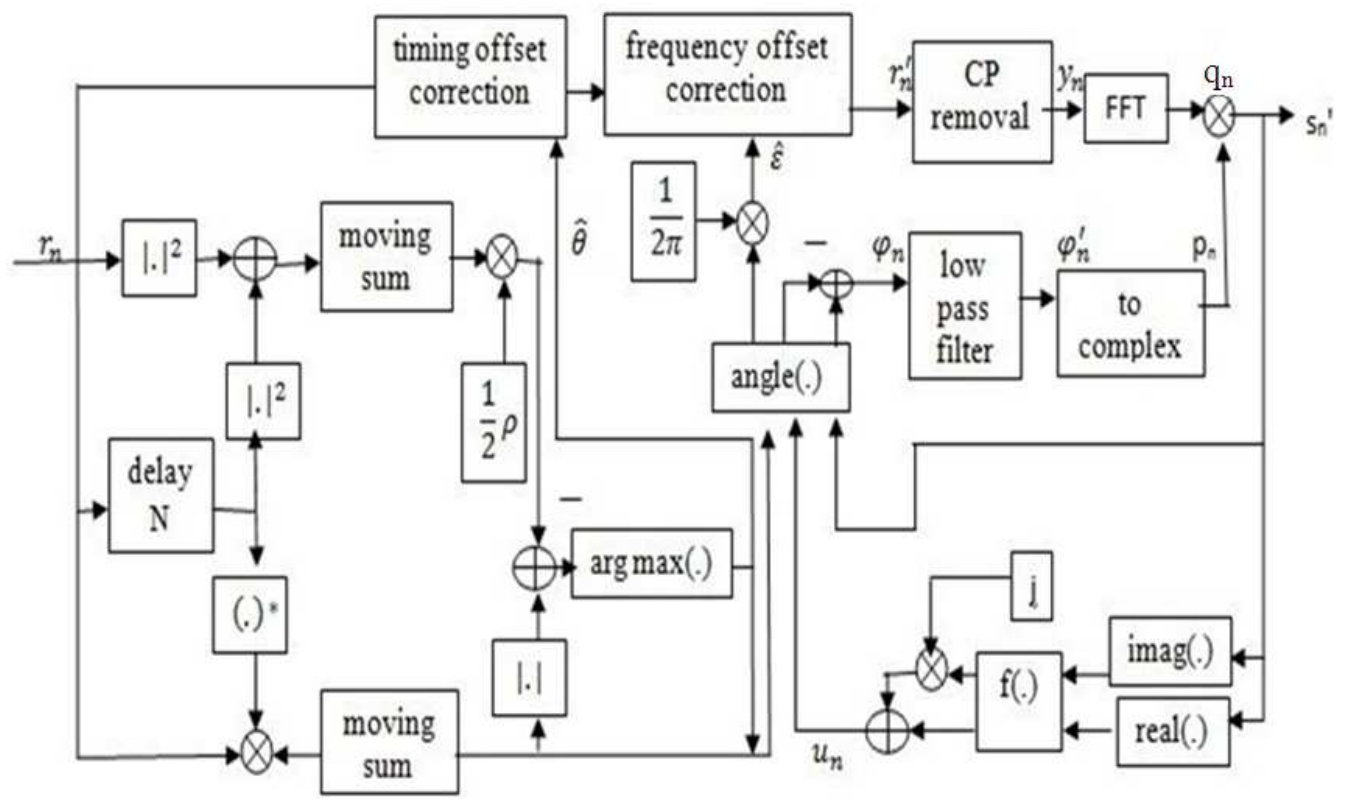

Fig. 2. Structure of frequency offset correction scheme

$$
\begin{aligned}
& \Phi(\mathrm{m}) \underline{\Delta} \frac{1}{2} \sum_{\mathrm{k}=\mathrm{m}}^{\mathrm{m}+\mathrm{L}-1}|\mathrm{r}(\mathrm{k})|^{2}+|\mathrm{r}(\mathrm{k}+\mathrm{N})|^{2} \\
& \rho \Delta\left|\frac{\mathrm{E}\left\{\mathrm{r}(\mathrm{k}) \mathrm{r}^{*}(\mathrm{k}+\mathrm{N})\right\}}{\sqrt{\mathrm{E}\left\{|\mathrm{r}(\mathrm{k})|^{2}\right\} \mathrm{E}\left\{|\mathrm{r}(\mathrm{k}+\mathrm{N})|^{2}\right\}}}\right|
\end{aligned}
$$

where, $\rho$ is the magnitude of the correlation coefficient between $\mathrm{r}(\mathrm{k})$ and $\mathrm{r}(\mathrm{k}+\mathrm{N})$ Equation (19):

$$
\rho=\frac{\sigma_{\mathrm{s}}^{2}}{\sigma_{\mathrm{s}}^{2}+\sigma_{\mathrm{n}}^{2}}=\frac{\mathrm{SNR}}{\operatorname{SNR}+1}
$$




$$
\begin{aligned}
& \hat{\theta}_{\mathrm{ML}}=\arg \max \{|\gamma(\theta)|-\rho \Phi(\theta)\} \\
& \hat{\varepsilon}_{\mathrm{ML}}=-\frac{1}{2 \pi} \angle \gamma\left(\hat{\theta}_{\mathrm{ML}}\right)
\end{aligned}
$$

And the effective signal-to-noise ratio SNR due to both additive noise and ICI is shown to be lower bounded by Equation (22):

$$
\operatorname{SNR}_{\mathrm{e}}(\varepsilon)=\frac{\mathrm{SNR}}{1+0.5947 \mathrm{SNR} \cdot \sin ^{2} \pi \varepsilon}\left(\frac{\sin \pi \varepsilon}{\pi \varepsilon}\right)
$$

The analytical BER Equation (23) with BPSK in the presence of Gaussian noise is given by:

$$
\mathrm{BER} \approx \mathrm{Q}\left(\sqrt{\frac{\frac{\sigma_{\mathrm{X}}^{2} \sin ^{2} \pi \varepsilon}{\sin ^{2} \frac{\pi \varepsilon}{M}} \cos ^{2} \frac{\pi \varepsilon(\mathrm{M}-1)}{\mathrm{M}}}{\sigma_{\mathrm{x}}^{2}\left(1-\frac{\sin ^{2} \pi \varepsilon}{\mathrm{M}^{2} \sin ^{2} \frac{\pi \varepsilon}{M}}\right)+\sigma_{\mathrm{n}}^{2}}}\right)
$$

The received signal $r_{n}$ is sent to the ML estimator and then both the integer frequency offset $\theta$ and fractional frequency offset $\varepsilon$ are given. After the timing and frequency offset correction, $\mathrm{CP}$ is removed from $r_{n}$ and the remaining signal $r_{n}$ of length $N$ is given to the FFT block as input. The FFT output $y_{n}$ is multiplied by the complex signal $p_{n}$ to generate the signal $\mathrm{q}_{\mathrm{n}}$ for QAM demodulation and $\mathrm{q}_{\mathrm{n}}$ is divided into the real and imaginary parts and then both are sent to the phase difference generator which has the transfer function of $\mathrm{f}(\mathrm{x})$ in 16 QAM. $\mathrm{f}(\mathrm{x})$ is given by $(\mathrm{n}=3)$ Equation (24):

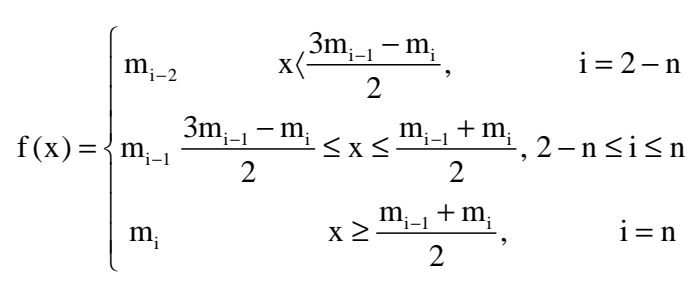

where, $\mathrm{m}_{\mathrm{i}}(\mathrm{i}= \pm 1, \pm 2, \pm 3$ ) is the $\mathrm{x}$-coordinate or $\mathrm{y}$ coordinate value of the QAM constellation point and the sign of is i gnifies the sign of the coordinate value. After that the output of imaginary part is multiplied by $\mathrm{j}$ and adds to the output of real part to form the signal $u_{n}$ Equation (25):

$$
\mathrm{u}_{\mathrm{n}}=\operatorname{real}\left(\mathrm{f}\left(\mathrm{q}_{\mathrm{n}}\right)\right)+\mathrm{j} \cdot \operatorname{imag}\left(\mathrm{f}\left(\mathrm{q}_{\mathrm{n}}\right)\right)
$$
by:

The phase difference $\varphi_{\mathrm{n}}$ Equation (26) is generated

$$
\phi_{\mathrm{n}}=\operatorname{angle}\left(\mathrm{q}_{\mathrm{n}}\right)-\operatorname{angle}\left(\mathrm{u}_{\mathrm{n}}\right)
$$

A low-pass filter is used to eliminate the noise in $\varphi_{\mathrm{n}}$ and output the signal $\varphi_{\mathrm{n}}$, which is the phase difference between the received signal and it's most proximate real value. $\varphi_{\mathrm{n}}$ could not be used to fix the phase error Equation (27) directly and it needs a angle to complex conversion:

$\mathrm{p}_{\mathrm{n}}=\cos \left(\phi_{\mathrm{n}}^{\prime}\right)+\mathrm{j} \cdot \sin \left(\phi_{\mathrm{n}}^{\prime}\right)$

\section{NUMERICAL RESULTS}

As expected, all results show that BER increases with increase in frequency offsets. The effect of Normalized CFO gives the BER performance of BPSK modulation scheme as illustrated in Fig. 3-5. These show that BER increases with increase in frequency offset.

Figure 3 shows the performance of BER vs. frequency offset over a rayleigh fading channel. Here, for the no fading case, BER increases from 0.0013 to 0.4 with increase in frequency offset in $(-0.5,0.5)$. For the rayleigh fading case, BER increases from 0.0011 to 0.7 with increase in the frequency offset in $(-0.5,0.5)$.

Figure 4 shows the performance of BER vs. frequency offset over Weibull fading channel. Here, the BER increases from 0.013 to 0.023 with increase in frequency offset $(-0.5,0.5)$.

Figure 5 shows the performance of the BER versus frequency offset over a Nakagami fading channel. Here, the BER increases from 0.07 to 0.12 with increase in frequency offset in $(-0.5,0.5)$.

The following graphs show the performance of the system due to the effect of CFO. Figure 6 shows the SNR Vs BER for BPSK modulation under the effect of various values of $\mathrm{CFO}$ with $\mathrm{N}=256, \mathrm{CP}=16$.

Figure 7 shows the effect of CFO in MIMO-OFDM systems with 16 QAM modulation with $\mathrm{N}=256, \mathrm{CP}=16$ under the effect of various values of CFO. 
Sabitha Gauni and Kumar Ramamoorthy / Journal of Computer Science 10 (2): 198-209, 2014

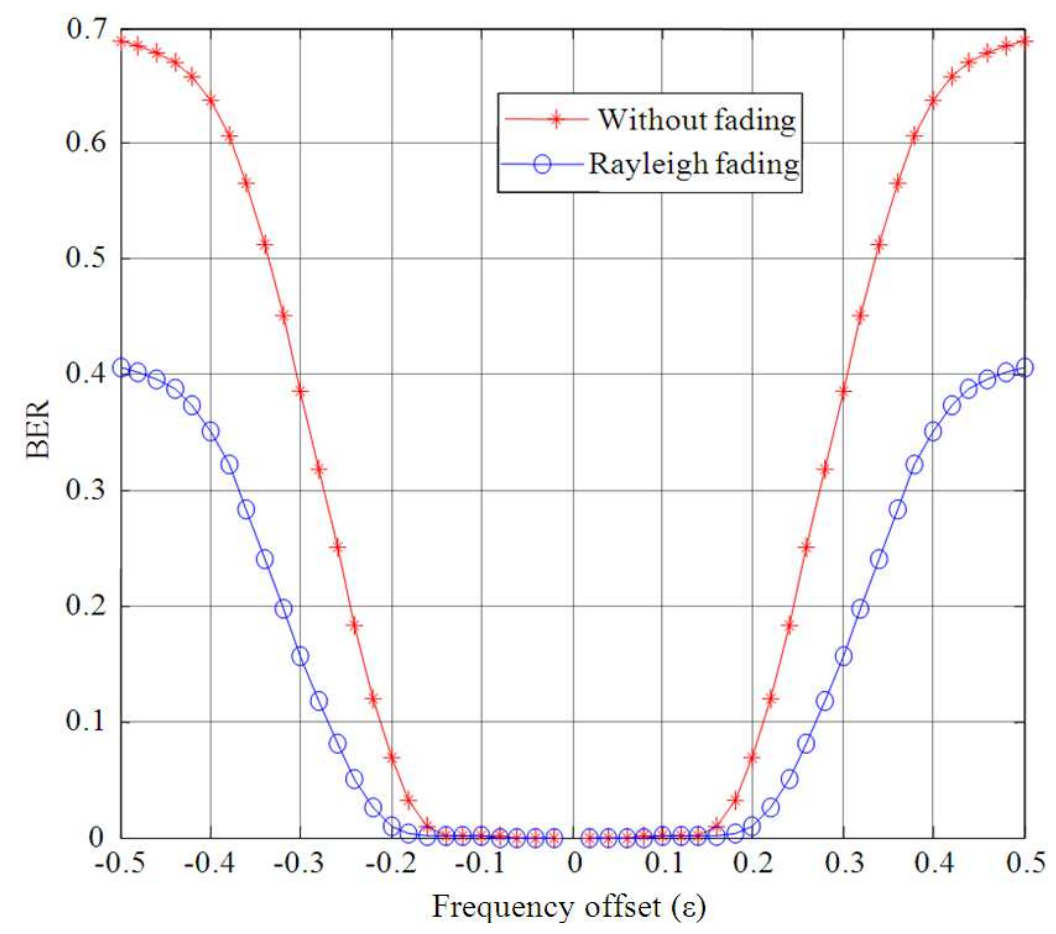

Fig. 3. BER Vs frequency offset with Gaussian distribution over a rayleigh fading channel

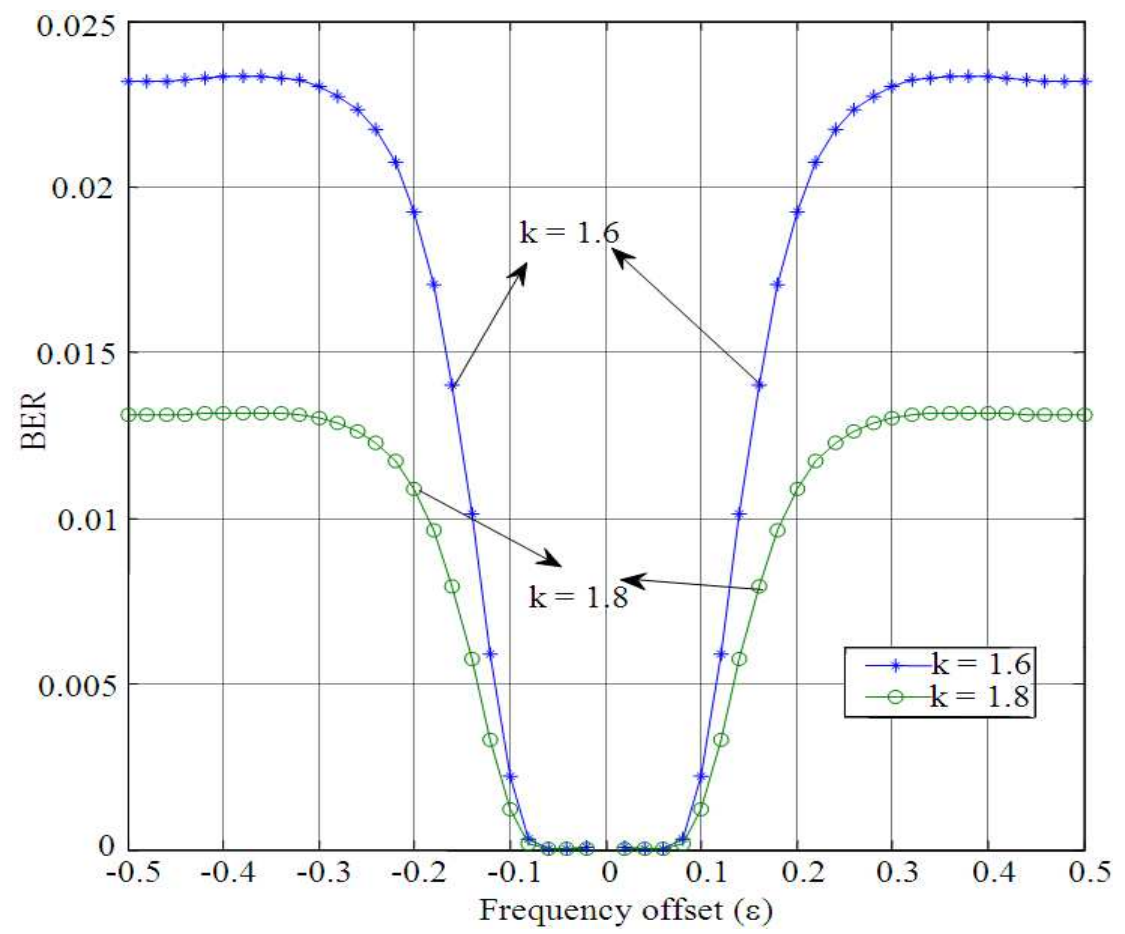

Fig. 4. BER Vs frequency offset with Gaussian distribution over a Weibull fading channel 
Sabitha Gauni and Kumar Ramamoorthy / Journal of Computer Science 10 (2): 198-209, 2014

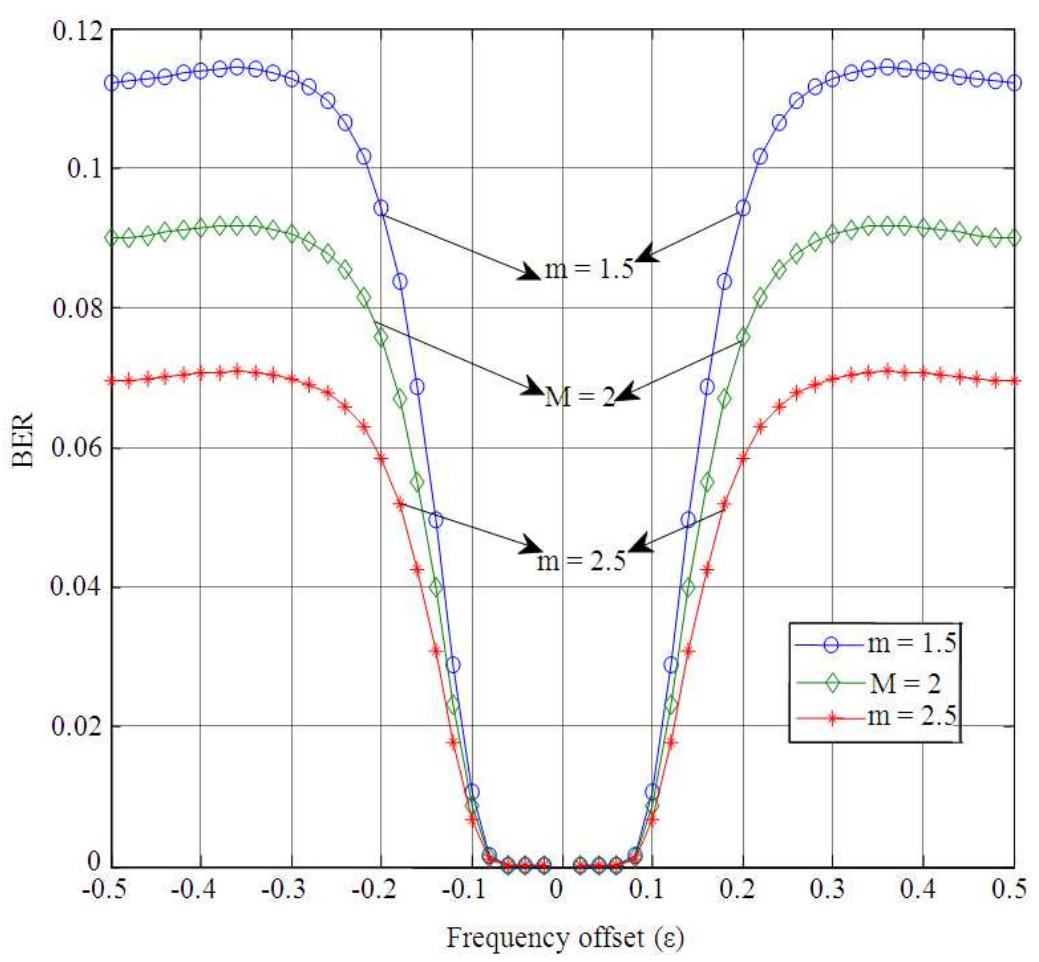

Fig. 5. BER Vs. Frequency offset with Gaussian distribution over a Nakagami fading channel

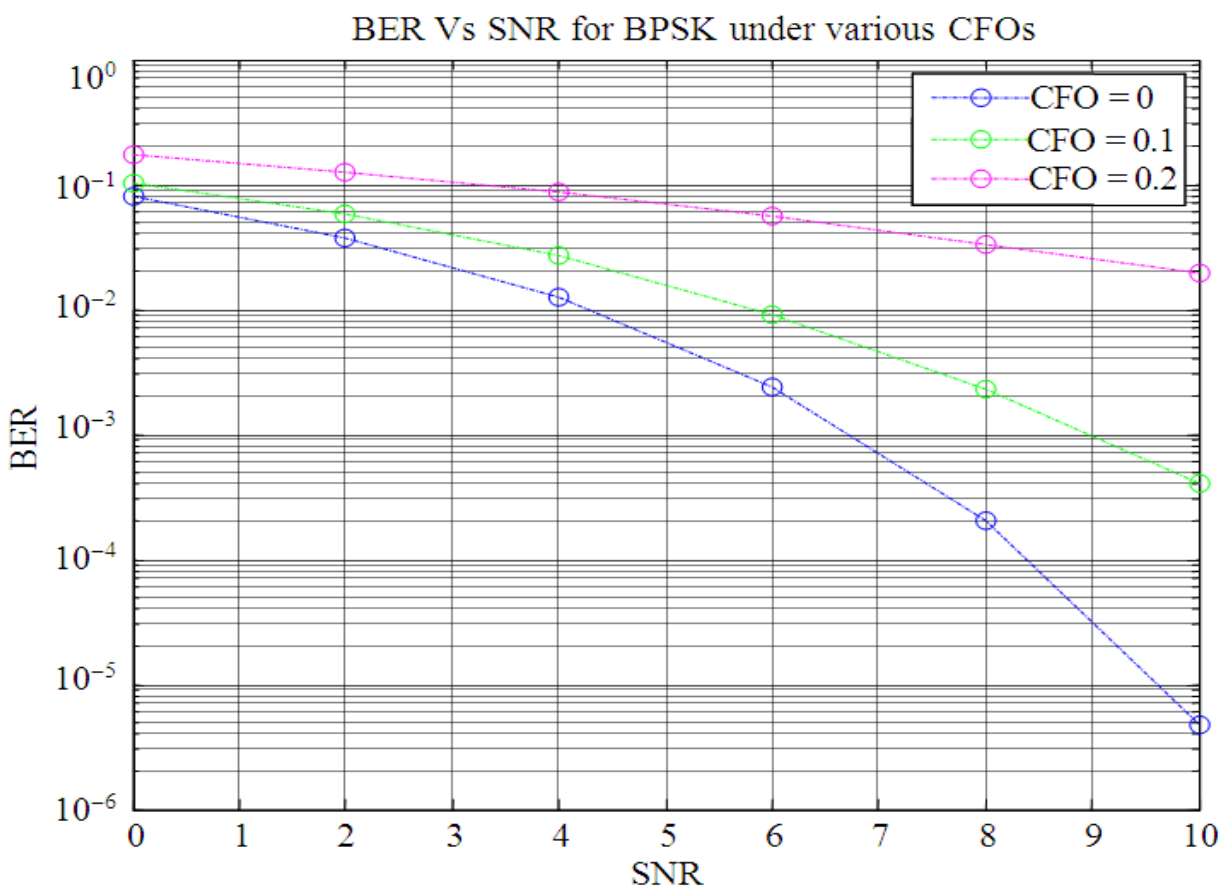

Fig. 6. BER Vs SNR for MIMO-OFDM system with BPSK modulation under various frequency offsets 
Sabitha Gauni and Kumar Ramamoorthy / Journal of Computer Science 10 (2): 198-209, 2014

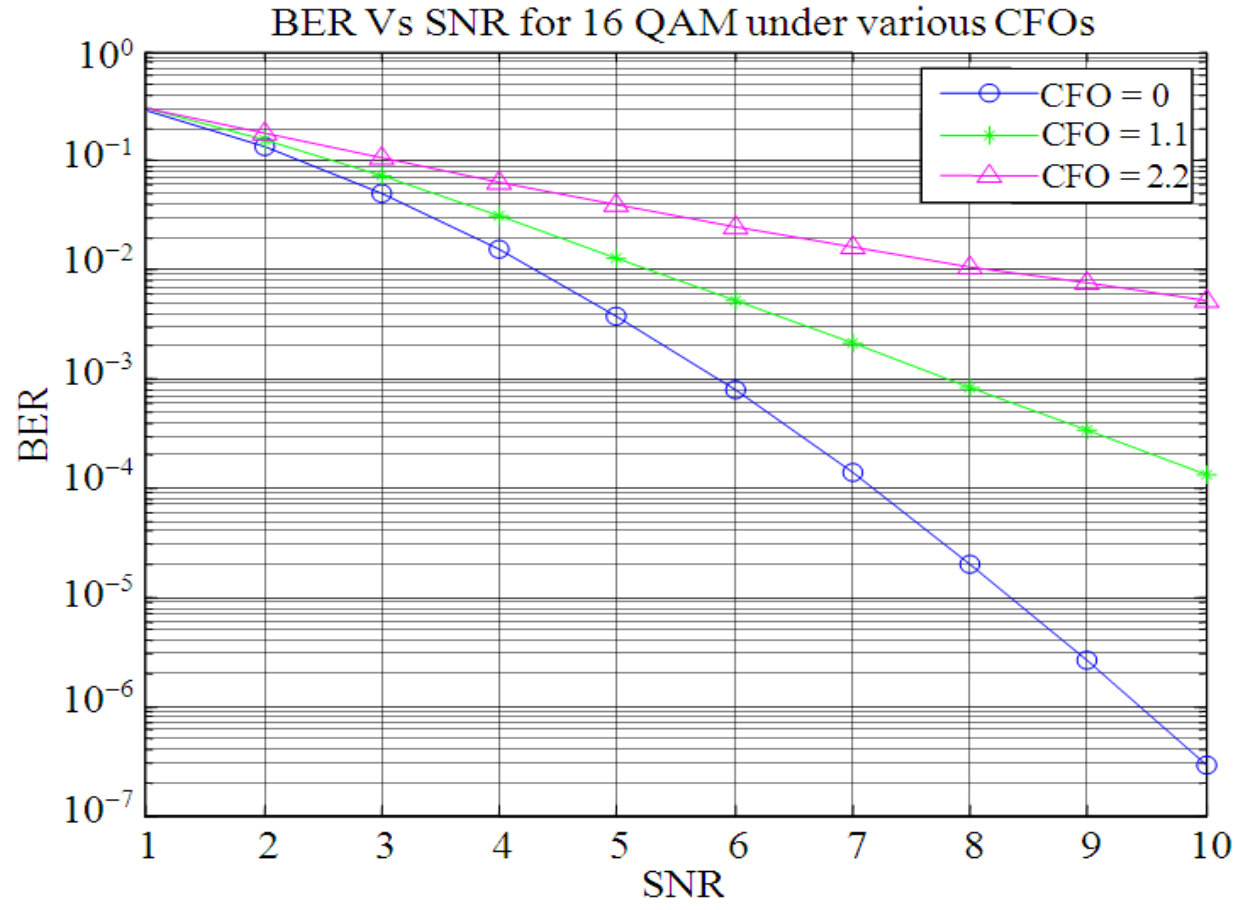

Fig. 7. BER verses SNR for MIMO-OFDM system with various frequency offsets

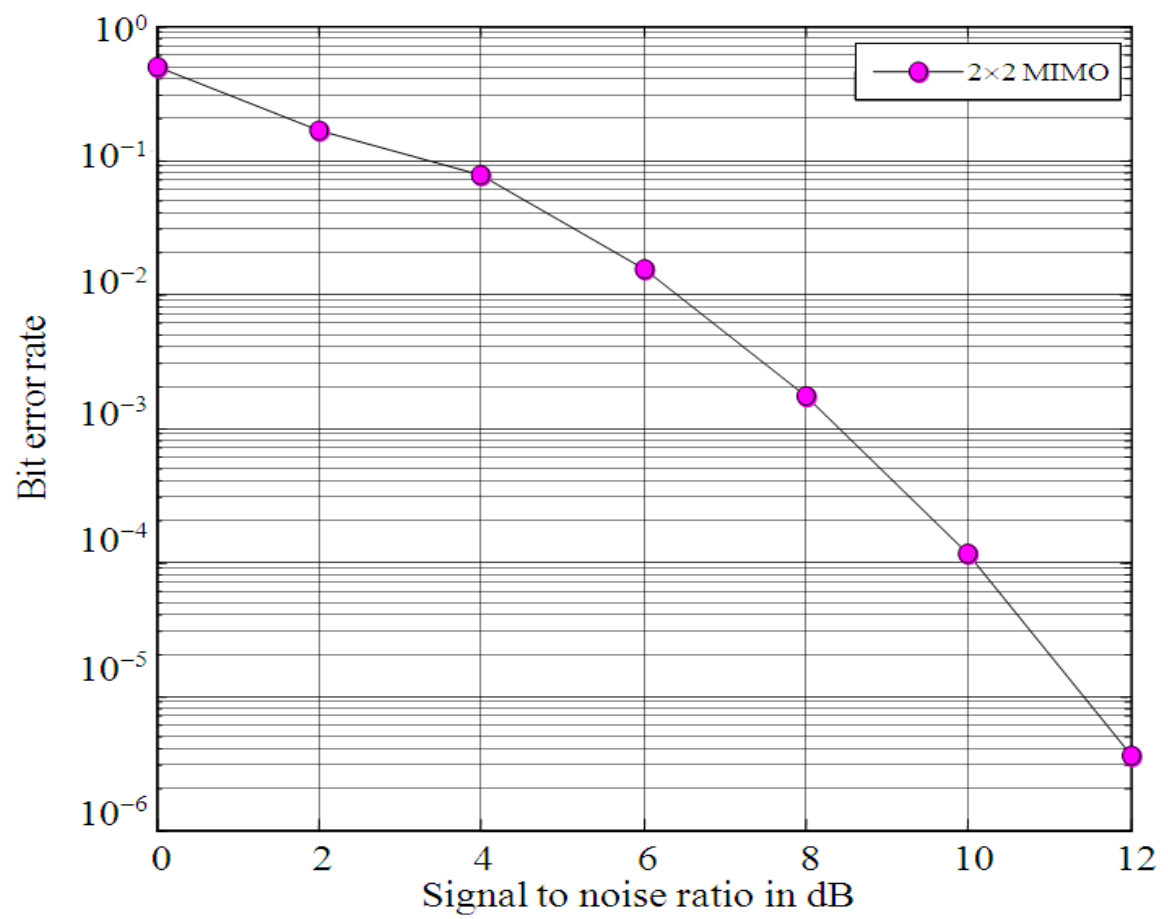

Fig. 8. BER verses SNR for $2 \times 2$ MIMO-OFDM system for rayleigh channel 
Sabitha Gauni and Kumar Ramamoorthy / Journal of Computer Science 10 (2): 198-209, 2014

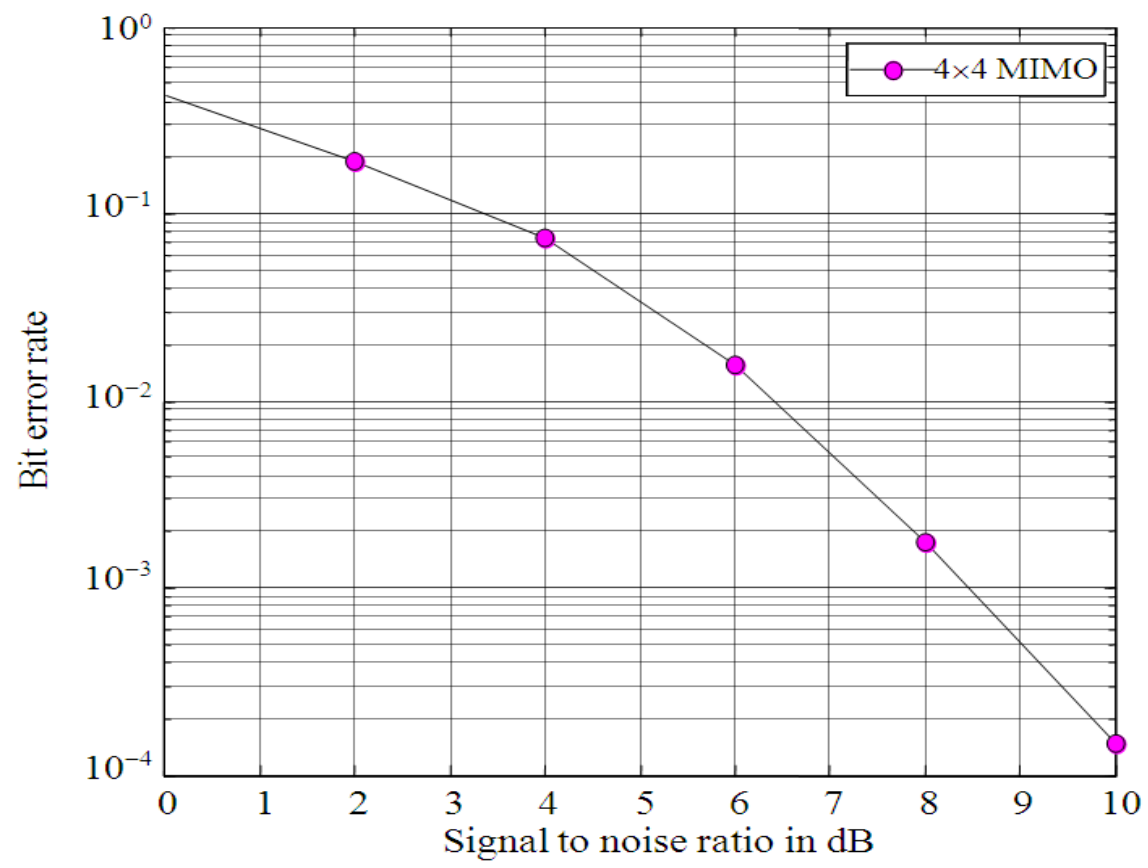

Fig. 9. BER verses SNR for 4×4 MIMO-OFDM system

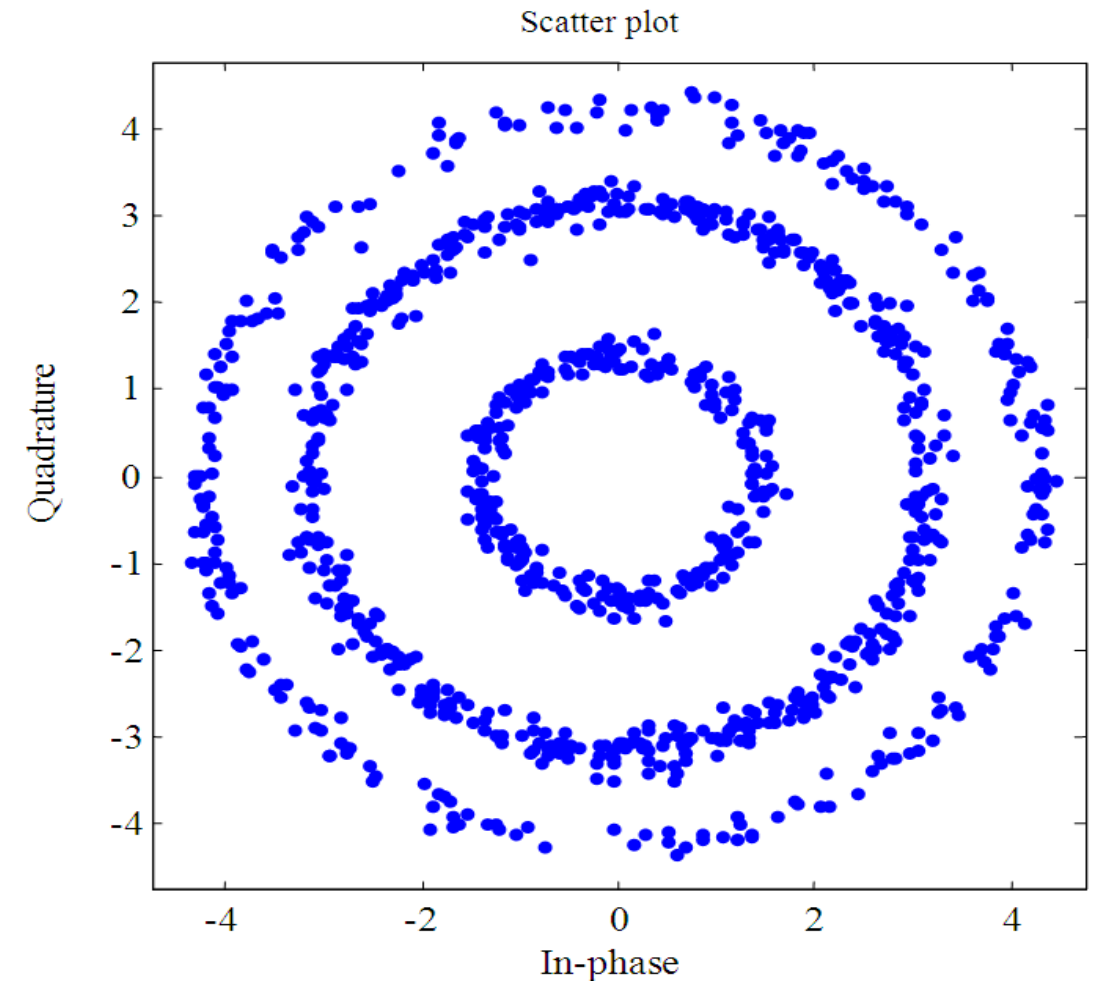

Fig. 10. Signal constellation at frequency offset $\varepsilon=0.01$, without correction at the receiver 


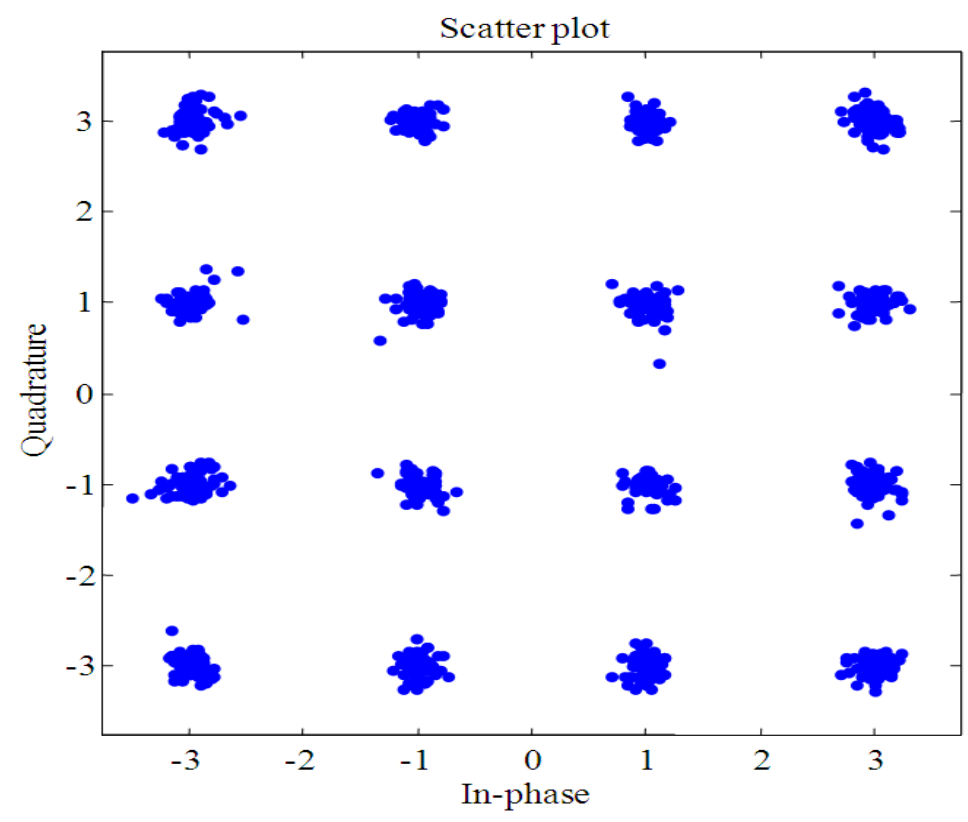

Fig. 11. Signal constellation at frequency offset $\varepsilon=0.01$ after processing by the proposed scheme at the receiver

The performance of the proposed scheme is shown in Fig. 8 and 9 with $2 \times 2$ MIMO-OFDM and $4 \times 4$ MIMOOFDM systems for 16QAM with $\mathrm{N}=1024, \mathrm{CP}=128$ and $\varepsilon=0.01$. The bit error rate is much same at low SNR values and for high SNR values this scheme is more sensitive to frequency offset. The bit error rate for $2 \times 2$ MIMO-OFDM with $\varepsilon=0.01$ is shown in Fig. 11 and the bit error rate for $4 \times 4$ MIMO-OFDM with $\varepsilon=0.01$ shown in Fig. 9 .

The 16-QAM constellation without and with the proposed scheme is shown in Fig. 10-11 respectively. Here phase offset is proportional to the sampling time offset and subcarrier index ' $\mathrm{k}$ ', forcing the signal constellation to be rotated around the origin, shown in Fig. 10. Therefore, the effect of all the accumulated frequency offset if not corrected will result in difficulty to map the constellation points. There are heavy chances of chaos in the identification of the symbols. After processing the frequency offset by the proposed scheme, the constellation point is identified easily, as shown in Fig. 11.

\section{CONCLUSION}

In the study, we have analyzed the performance of an OFDM system using BPSK modulation with frequency offsets over various fading channels. It can be observed that system performance severely degrades with increase in ICI due to the offsets. The analytical expressions obtained closely match the numerical results. The simulated results show that as the frequency offsets in Ray Leigh, We bull and Nakagami fading channels increases, the BER also increases. The reported BER can be further reduced by using channel estimation.

A method for frequency offset estimation and correction scheme for MIMO-OFDM system is proposed. The proposed scheme uses the ML estimator for estimation of the CFO in a MIMO OFDM system and the remaining block is used for tracking. The system performance was analyzed with an AWGN channel. The simulation results show that the frequency offsets caused by sampling clock in MIMO-OFDM receiver are corrected using the proposed scheme. Thus reduction in the implementation complexity and thereby reduction in the computational cost is acheived.

\section{REFERENCES}

Ahmed, A.O.M., H.S. Al-Kutubi and N.A. Ibrahim, 2010. Comparison of the Bayesian and maximum likelihood estimation for weibull distribution. J. Math. Stat., 6: 100-104. DOI: 10.3844/jmssp.2010.100.104

Gauni, S., J. Elakkiya, R. Kumar and V. Bhaskar, 2012. Performance analysis of OFDM systems with Gaussian distributed phase and frequency offsets. Int. J. Comput. Appli., 47: 42-49. DOI: $10.5120 / 7155-9864$ 
Kumaran, V.N.S., S. Rajkumar and S.J. Thiruvengadam, 2013. Performance analysis of orthogonal frequency division multiplexing based bidirectional relay network in the presence of phase noise. Am. J. Applied Sci., 10: 1335-1344. DOI: 10.3844/ajassp.2013.1335.1344

Qatawneh, I.A.Z., 2013. Multiple-input multiple-output transmission over a multipath faded channels. Am. J. Applied Sci., 10: 230-238. DOI: 10.3844/ajassp.2013.230.238
Suresh, M.N., S.J. Thiruvengadam and V. Abhaikumar, 2012. Symbol timing estimation in multiband orthogonal frequency division multiplexing based ultrawide band system. Am. J. Applied Sci., 9: 505509. DOI: 10.3844/ajassp.2012.505.509

Ullah, M.H., M.T. Islam, M. Sing, R. Nordin and B. Bais, 2012. Design of transmitter for CDM based $2 \times 2$ multiple input multiple output channel sounder for multipath delay measurement. Am. J. Applied Sci., 9: 117-122. DOI: 10.3844/ajassp.2012.117.122 